\title{
BMJ Open What predicts regression from pre- diabetes to normal glucose regulation following a primary care nurse- delivered dietary intervention? A study protocol for a prospective cohort study
}

\author{
Kirsten Coppell (D) , ${ }^{1}$ Trish Freer, ${ }^{2}$ Sally Abel, ${ }^{3}$ Lisa Whitehead, ${ }^{4}$ \\ David Tipene-Leach, ${ }^{5}$ Andrew R Gray, ${ }^{6}$ Tony Merriman, ${ }^{7}$ Trudy Sullivan, ${ }^{8}$ \\ Jeremy Krebs, ${ }^{9}$ Leigh Perreault ${ }^{10}$
}

To cite: Coppell K, Freer T, Abel S, et al. What predicts regression from pre-diabetes to normal glucose regulation following a primary care nursedelivered dietary intervention? A study protocol for a prospective cohort study. BMJ Open 2019;9:e033358. doi:10.1136/ bmjopen-2019-033358

- Prepublication history for this paper is available online. To view these files, please visit the journal online (http://dx.doi. org/10.1136/bmjopen-2019033358).

Received 31 July 2019 Revised 07 November 2019 Accepted 19 November 2019

Check for updates

(C) Author(s) (or their employer(s)) 2019. Re-use permitted under CC BY-NC. No commercial re-use. See rights and permissions. Published by BMJ.

For numbered affiliations see end of article.

Correspondence to

Dr Kirsten Coppell;

kirsten.coppell@otago.ac.nz

\section{ABSTRACT}

Introduction Pre-diabetes is a high-risk state for the development of type 2 diabetes mellitus (T2DM) and cardiovascular disease. Regression to normoglycaemia, even if transient, significantly reduces the risk of developing T2DM. The primary aim of this mixed-methods study is to determine if there are clinically relevant differences among those with pre-diabetes and excess weight who regress to normoglycaemia, those who have persistent pre-diabetes and those who progress to T2DM following participation in a 6-month primary care nursedelivered pre-diabetes dietary intervention. Incidence of T2DM at 2 years will be examined.

Methods and analysis Four hundred participants with pre-diabetes (New Zealand definition glycated haemoglobin $41-49 \mathrm{mmol} / \mathrm{mol}$ ) and a body mass index $\geq 25 \mathrm{~kg} / \mathrm{m}^{2}$ will be recruited through eight primary care practices in Hawke's Bay, New Zealand. Trained primary care nurses will deliver a 6 -month structured dietary intervention, followed by quarterly reviews for 18 months post-intervention. Clinical data, data on lifestyle factors and health-related quality of life (HR-QoL) and blood samples will be collected at baseline, 6 months, 12 months and 24 months. Sixty participants purposefully selected will complete a semi-structured interview following the 6-month intervention. Poisson regression with robust standard errors and clustered by practice will be used to identify predictors of regression or progression at 6 months, and risk factors for developing T2DM at

2 years. Qualitative data will be analysed thematically. Changes in HR-QoL will be described and potential cost savings will be estimated from a funder's perspective at 2 years.

Ethics and dissemination This study was approved by the Northern A Health and Disability Ethics Committee, New Zealand (Ethics Reference: 17/NTA/24). Study results will be presented to participants, published in peer-reviewed journals and presented at relevant conferences.

Trial registration number ACTRN12617000591358; Pre-results.

\section{Strengths and limitations of this study}

- This mixed-methods study follows a pragmatic nonrandomised pilot study which showed that a primary care nurse-delivered 6-month dietary intervention for people with pre-diabetes and body mass index $\geq 25 \mathrm{~kg} / \mathrm{m}^{2}$ was feasible in a primary care setting, and weight loss was achieved in the intervention group.

- The study is set in a geographical region where approximately $21 \%$ of the population is Māori, the indigenous peoples of New Zealand, who have high rates of pre-diabetes and type 2 diabetes mellitus.

- Following the 6-month dietary intervention, participants will be reviewed every 3 months by primary care nurses for 18 months.

- A possible limitation is that the study is taking place in a real-world setting, and health sector changes influencing the primary care environment may influence the study outcomes.

- This study is limited as it will not include a control group.

\section{INTRODUCTION}

The prevalence of diabetes and concomitant costs continue to increase. ${ }^{1-4}$ Diabetes now affects an estimated $8.8 \%$ of adults globally. ${ }^{5}$ Pre-diabetes, a condition in which blood glucose levels are higher than normal, but not high enough to be classified as diabetes, is more common, affecting as many as $30 \%-50 \%$ of some adult populations. ${ }^{6-9}$ Without treatment, about $5 \%-10 \%$ of those with pre-diabetes will progress to type 2 diabetes mellitus (T2DM) annually, and most will eventually develop T2DM. ${ }^{10}{ }^{11}$ Lifestyle modification and weight loss can prevent or delay progression, ${ }^{12-18}$ with the overall risk being approximately halved. ${ }^{19}$ Medication 
has also been shown to prevent T2DM in people with prediabetes enrolled in clinical trials. ${ }^{12}$ 19-21

\section{Implementing diabetes prevention lifestyle interventions in primary care: the challenges}

Implementing diabetes prevention lifestyle clinical trial evidence into real-world settings is challenging, particularly in primary care settings, ${ }^{22-24}$ where those with pre-diabetes are typically a more heterogeneous group compared with those participating in clinical trials. ${ }^{25}$ Further, the cost of delivering lifestyle interventions in primary care can be a barrier. ${ }^{26}$ The Diabetes Prevention Program (DPP) lifestyle intervention costs US $\$ 1399$ per participant in the first year. ${ }^{27}$ A significant proportion of this cost was specialist lifestyle intervention staff, who are often employed in diabetes prevention lifestyle programmes, irrespective of the setting. ${ }^{12-17}{ }^{28-35}$ Few studies have specifically employed primary care nurses to deliver lifestyle advice ${ }^{36-39}$ although dietary advice given by an appropriately trained nurse can be as effective as that given by a dietitian in the primary care setting, ${ }^{38}$ and is potentially more sustainable and cost-effective. ${ }^{40}$

Furthermore, as pre-diabetes increases the risk of not only T2DM, but also cardiovascular disease, ${ }^{61141}$ and the microvascular complications associated with diabetes, ${ }^{642}$ the goal of pre-diabetes screening and treatment should arguably be regression to normoglycaemia. ${ }^{11} 2143$ Even if transient, regression to normoglycaemia is associated with a significantly reduced risk of developing T2DM, independent of whether this occurs spontaneously or in response to lifestyle advice or metformin therapy. ${ }^{44}$ Greater insulin secretion, lower baseline glucose concentrations, younger age, weight loss and intensive lifestyle modification predict restoration of normoglycaemia, ${ }^{45-47}$ but other factors such as sociocultural, economic and health service delivery, which have not been previously examined, may also be important.

\section{Pre-diabetes lifestyle interventions have been implemented in primary care}

The primary care-based Pre-diabetes Intervention Package (PIP) is a feasible primary care nurse-delivered 6-month pre-diabetes lifestyle intervention with a focus on diet, sociocultural context and goal setting, for overweight and obese patients with pre-diabetes. ${ }^{48}$ In this pragmatic mixed-methods non-randomised pilot study conducted in the Hawke's Bay region, New Zealand (NZ), intervention implementation fidelity was high and after adjustment, the intervention group lost a mean $1.3 \mathrm{~kg}$ more than the control group $(\mathrm{p}<0.0 .001)$. The mean glycated haemoglobin (HbA1c) decreased in the intervention group and increased in the control group, but the difference was not significantly different. Extending the study and offering the intervention to all study practices, alongside exploring clinical, as well as sociocultural, economic and genetic variables, provides an opportunity to determine if there are clinically relevant differences among those with pre-diabetes who regress to normoglycaemia, who have persistent pre-diabetes and who progress to T2DM. It is anticipated that these prospectively collected data will contribute to limited international data on sociocultural factors that may be associated with regression from prediabetes to normoglycaemia, ${ }^{45}$ and to clinical decisionmaking risk assessments in the primary care setting. ${ }^{2149}$

The primary aim of this study is (1) to determine if there are clinically relevant differences among those with pre-diabetes who regress to normoglycaemia, those who have persistent pre-diabetes and those who progress to T2DM at 6 months following participation in a structured primary care nurse-delivered pre-diabetes dietary intervention in the primary care setting. The secondary aims are (2) to quantify the reduction in incident T2DM at 2 years in participants who regressed to normoglycaemia at 6 months compared with the other participants; (3) to qualitatively explore and examine barriers, challenges and facilitators of clinically meaningful lifestyle changes between those who regress to normoglycaemia and those who do not; (4) to explore whether a T2DM genetic risk score of common genetic variants might influence regression to normoglycaemia at 2 years in a sub-sample, (5) to describe changes in HR-QoL at 2 years and (6) to estimate the potential cost savings to the health sector associated with a reduction in T2DM.

\section{METHODS AND ANALYSIS}

This extension of the PIP pilot study is a mixed-method prospective cohort study with pre-intervention and postintervention measures and a qualitative study. ${ }^{50} 51$

\section{NZ context}

In NZ, among adults aged 15 years and over, the prevalence of diabetes is $7.0 \%$ and the prevalence of pre-diabetes is $25.5 \% .^{7}$ Māori, the indigenous people of NZ, and Pacific people have higher rates than the NZ European and Other ethnic group, with diabetes rates of $9.8 \%, 15.4 \%$ and $6.1 \%$, respectively, and pre-diabetes rates of $30.4 \%$, $29.8 \%$ and $24.6 \%$, respectively. ${ }^{7}$ Screening for diabetes and pre-diabetes is recommended as part of cardiovascular risk assessment. ${ }^{52}$ For those with no symptoms and no known risk factors, screening is recommended from 45 years for men and 55 years for women, with Māori, Pacific, Indo-Asian peoples, and those with known cardiovascular risk factors or at high risk of developing diabetes to be screened 10 years earlier, or even younger if there is particular clinical concern regarding unfavourable risk factors. ${ }^{52}$

\section{Study setting}

The study will be conducted in general practices in the urban zones of two neighbouring provincial cities (Napier and Hastings) in the Hawke's Bay region, NZ. Napier had a population of 57240 (18.2\% Māori) and Hastings District had a population of 73245 (23.0\% Māori) in 2013. ${ }^{53}$ Māori comprise $15 \%$ of the NZ population. ${ }^{54}$ The general practices will be members of the Health 
Hawke's Bay Primary Health Organisation (PHO). PHOs are not-for-profit organisations that provide primary health services either directly or through their provider members. Health Hawke's Bay is the only PHO in the study region.

\section{Patient and public involvement}

The PIP was designed in collaboration with the Health Hawke's Bay PHO. ${ }^{48}$ The intervention was first pre-tested with members of the public, then primary care nurses reviewed and contributed to the implementation and research protocols at a meeting. The process evaluation by an independent researcher ${ }^{48}$ enabled views about the intervention and its implementation from patient participants, participating nurses and Health Hawke's Bay PHO staff involved in the design and implementation to be incorporated into the planning of this study. Results of the pilot study and process evaluation were presented and discussed at a forum to which all participants (patients and professionals) were invited.

\section{Participant eligibility}

General practices who use the Medtech patient management system and employ primary care nurses will be invited to participate in the study. The reason for including general practices using Medtech is that this is the dominant patient management system in Hawke's Bay, and study data collection systems had been modified for Medtech only in the pilot study. The demographic description of practice populations will be reviewed to ensure that patients with different sociocultural backgrounds are offered the opportunity to participate.

Adults aged 18-69 years with pre-diabetes (defined as HbAlc 41-49 $\mathrm{mmol} / \mathrm{mol}$ according to the NZ diagnostic criteria) $)^{52}$ and a body mass index $(\mathrm{BMI}) \geq 25 \mathrm{~kg} / \mathrm{m}^{2}$ will be included. Pre-diabetes will have been diagnosed following screening (at the time of a recommended cardiovascular risk assessment or because they are deemed to be at risk) ${ }^{52}$ We set a lower age limit of 18 years to reflect the high prevalence of pre-diabetes in young adults in NZ, particularly in high-risk ethnic groups including indigenous Māori and Pacific. ${ }^{7}$ Those with a history of diabetes, prescribed metformin, unable to communicate in English, with a terminal illness or planning to move from the area during the first 6 months of the study and women pregnant at the time of study enrolment will be excluded.

\section{Identification of potential participants and recruitment}

General practice patient management systems will be used to identify potential eligible participants. Queries will be conducted using the HbAlc, BMI, age and metformin status criteria to generate lists of potential participants. The queries will not be time-constricted. Primary care nurses will review these lists to check eligibility status including co-morbidities, current medications, confirmation of English-speaking ability and pregnancy status. The lists will be generated in no particular order.
Potential participants will be recruited sequentially according to the random lists generated, in order to minimise selection bias. The research nurse will check these lists regularly to assess who has been invited, who has declined and who is yet to be invited. A letter inviting participation and information about the study will be sent to potential participants in blocks to manage nurse's workload. Potential participants will be contacted by their primary care nurse 5-7 days later to determine participation status and to make a baseline study appointment for those choosing to participate. For those who decline to participate, the nurse will record the reason(s). Those diagnosed with pre-diabetes after recruitment begins and those who meet the eligibility criteria will be invited to participate at the time of their diagnosis.

\section{Intervention}

The intervention will be delivered by trained primary care nurses in the general practice setting. The key underpinning principle of the intervention is to provide participants (and their family) with an understanding of the principles of healthy eating and to deliver consistent evidence-based dietary messages, so they are empowered to make good dietary choices; that is, the dietary advice will not be prescriptive. This pragmatic intervention package has six components ${ }^{48}$ :

\section{Health Professional Training and Support}

Primary care nurses will undertake an intensive 3-4hour theoretical and practical training course. The course content will include the rationale for the study, key nutritional concepts, dietary assessment in the primary care setting (using Starting the Conversation (STC) ${ }^{55}$ modified for the NZ context) ${ }^{48}$ healthy conversations and goal setting. The nurses will be instructed how to use the PIP study-structured dietary tool, ${ }^{48}$ and about the necessary research processes (informed consent and data collection). They will also be instructed on standard practices for measuring anthropometry and blood pressure. The course will be delivered by study investigators and a local dietitian. A training manual will provide reference material for both primary care nurses and participants, as well as research protocols. A half-day update course will be run at 6 months.

The local dietitian will be available by email and phone to answer any queries or discuss specific clinical cases. The dietitian will also arrange monthly clinical case review meetings for 6 months at each practice. Both the primary care nurses and dietitian found that dietitian's support and guidance was only required for up to 6 months in the pilot study. ${ }^{48}$ A research nurse will also visit participating practices to provide support and advice, as needed, to maximise intervention fidelity.

Individualised dietary assessment, goal setting and dietary advice sessions

The intervention goal is a $10 \%$ wt loss over 6 months. After providing informed written consent, participants 
will be offered an initial $30 \mathrm{~min}$ individualised dietary session with their primary care nurse. They will be encouraged to bring family to the session. A brief structured dietary assessment will be undertaken. STC:Diet, a validated eight-item simplified food frequency instrument designed for use in primary care and health-promotion settings, will be used. ${ }^{55}$ STC:Diet was minimally modified, with permission, for the NZ context. Specifically, the word 'sodas' was changed to 'soft drinks', and a traffic light system was added to indicate healthy, not-so healthy and unhealthy dietary habits. The nurse will review the STC:Diet responses; ask additional dietary prompt questions; seek additional contextual information, such as household membership and budget, who purchases household foods and specific dietary requirements/ choices such as vegetarianism and take anthropometric measures (height, weight and waist circumference) using calibrated equipment. The additional questions are called the Detailed Dietary Assessment (DDA). A 10\% wt loss goal over 6 months will be calculated. Responses to the STC:Diet and DDA will inform three dietary goals negotiated with the participant, and individualised dietary advice. Participants will be given the standard 'Be Active Every Day' pamphlet, which advises $30 \mathrm{~min}$ of physical activity of moderate intensity on most, if not all, days of the week. ${ }^{56}$ Follow-up intervention appointments will be arranged at 2-3 weeks, 6 weeks, 3 months, 4 months and 6 months, then 3-monthly appointments for a 'weigh-in' and provision of ongoing brief targeted dietary advice and support for 18 months. Intervention appointments at 6 weeks and 4 months were added, following feedback from both participants and nurses in the feasibility study. ${ }^{48}$ All study appointments will be at no cost to the participant.

\section{Key messages and consistent opportunistic reminders}

Each participant's three dietary goals will be recorded in the practice patient management system. These goals and accompanying clinical notes will facilitate opportunistic targeted advice and guidance by GPs, thus reinforcing dietary advice and support provided by the nurses. The goals will be reviewed and updated accordingly at follow-up nurse appointments.

\section{Nutritionally supportive primary care environment}

Prior to the beginning of this study, each intervention practice will be visited to discuss ways to enhance dietary messages provided by nurses. Specifically, the dietary information provided in pamphlets, magazines and posters in the waiting rooms will be reviewed and updated, if necessary, so dietary messages are appropriate and consistent. Provision of magazines that support reputable dietary messages and active living, hobbies and sports and posters promoting fruit and vegetables, such as those offered by Vegetables.co.nz (www.vegetables.co. $\mathrm{nz}$ ), will be encouraged.
Community-based group education for participants and their family At the 2-3week intervention appointment, nurses will refer participants to the Kia Ora programme, a community group nutrition education course consisting of six weekly sessions of 2.5 hours. The Kia Ora programme is an approved Stanford chronic disease self-management programme. ${ }^{5758}$ The education sessions will be delivered by lay trainers, and topics will include healthy eating, label reading, problem solving and making action plans.

\section{Written patient resources}

Readily available patient resources will be utilised. The key resource will be the Diabetes New Zealand booklet, Diabetes and healthy food choices, ${ }^{59}$ a clearly presented and easily understood source of dietary information. The booklet was used successfully in the LOADD study, ${ }^{60}$ and the PIP pilot study. ${ }^{48}$

\section{Data collection}

Data will be collected as part of the intervention appointments with primary care nurses, as was done in the pilot study. ${ }^{48}$ Study data collection forms have been incorporated into the Medtech patient management system. Additional data will be collected by a research nurse at separate study-specific visits. Recruitment began August 2017, and it is expected that data collection will be completed December 2021. The type and frequency of measurements are shown in table 1 . These include:

\section{Routine clinical data}

Clinical data will be collected by primary care nurses. These data will include demographics, medical history, family history, social history, brief dietary assessment, height, weight, waist circumference, blood pressure and bloods (HbA1c, total cholesterol, high-density lipoprotein cholesterol, triglycerides, liver enzymes (alanine aminotransferase, aspartate aminotransferase and gamma-glutamyl transferase) and urate. Known data such as date of birth and ethnicity will pre-populate the study data collection sheets and additional pre-diabetes clinical management data (eg, brief dietary assessment details) will be added by the primary care nurses during the intervention consultations.

\section{Additional study-specific quantitative data}

A research nurse will administer a questionnaire and arrange for a fasting blood sample to be taken. The questions will explore areas known to influence pre-diabetes lifestyle management. Data will be collected on selfmonitoring activities (self-weighing at home, food diaries and self-initiated attendance at support groups), social support systems, ${ }^{61}{ }^{62}$ sleep patterns, ${ }^{63}$ stages and processes in weight management, ${ }^{64} 65$ physical activity (the International Physical Activity Questionnaire (IPAQ) long) ${ }^{66}$ and HR-QoL using the EQ-5D. ${ }^{6768}$ The additional non-routine blood tests will include fasting insulin and fasting glucose (to enable calculation of homeostatic model assessment (HOMA) $)^{69}$ and the McAuley Index ${ }^{70}$ both measures 
of insulin resistance), and whole genome sequencing (WGS) in a sub-sample.

\section{Genetic testing}

The genomes from participants who, in addition to the main study, provide consent to genetic testing, and are from the opposite ends of the response spectrum will be sequenced, with the intention of sequencing genomes from all consenting participants as the cost of sequencing reduces. Using genome-wide single nucleotide polymorphism (SNP) genotyping platforms would not be adequate as these platforms use common genetic variants identified in other populations. WGS is the only practical approach as it will allow identification of uncommon (including Māori-specific) variants predicted to have a strong functional effect in the suite of genes to be analysed for a burden of functional variation. WGS also allows evaluation of regulatory variants which would not be possible using an exome sequencing approach. Sequencing will be done at 30 -fold coverage.

\section{Outcome measures}

The primary outcome measures will be HbAlc (mmol/ $\mathrm{mol}$ ) and weight $(\mathrm{kg})$. Other outcome measures will include waist circumference, BMI, social support score, behavioural change score and EQ-5D scores.

\section{Sample size calculations}

The sample size calculation used information from the Voglibose study. ${ }^{21}{ }^{71}$ It was assumed that $25 \%$ of those with pre-diabetes at baseline will regress to normoglycaemia at 6 months. Assuming 5\% loss to follow-up at 6 months, a sample of 400 people with pre-diabetes will provide $80 \%$ power to detect factors associated with regression at 6 months with relative risks for regression of 2.2 or higher, for any two groups each comprising between $20 \%$ and $80 \%$ of the sample using a two-sided test at $\mathrm{p}<0.05$ level of significance.

\section{Planned statistical analysis}

STROBE (Strengthening the Reporting of Observational Studies in Epidemiology) ${ }^{72}$ and TRIPOD (Transparent Reporting of a multivariable prediction model for Individual Prognosis Or Diagnosis) ${ }^{73}$ reporting standards will be used. Descriptive statistics will be provided for all measures of interest, as appropriate. For inferential analyses, all results will be reported showing effect sizes, $95 \%$ CIs and $\mathrm{p}$ values. Two-sided $\mathrm{p}<0.05$ will be considered statistically significant. Analyses will be conducted using R V.3.5.1 and Stata V.15.1 (or later versions).

Poisson regression with robust standard errors and clustered by practice (using Froot's extension to HuberWhite estimators ${ }^{74}$ ) will be used to identify predictors of regression to normoglycaemia and progression to T2DM at 6 months. Similar models will be used to examine risk factors for developing T2DM at 2years. Exploratory modelling will use Markov models to identify variables associated with transitions between the three glycaemic states of interest, defined according to the NZ definitions: 
normoglycaemia $(\mathrm{HbA} 1 \mathrm{c}<41 \mathrm{mmol} / \mathrm{mol})$, pre-diabetes $(41 \mathrm{mmol} / \mathrm{mol} \geq \mathrm{HbAl} \mathrm{c} \leq 49 \mathrm{mmol} / \mathrm{mol})$ and diabetes $(\mathrm{HbAlc}>49 \mathrm{mmol} / \mathrm{mol}) .^{52}$ Providing the number of transitions between each pair of states is sufficient, multistate models will initially be used to describe transition probabilities between these states over all measurement times (all three transitions are possible) with an absorbing state of death also reachable from any of these three states. Factors associated with transitions between these three glycaemic states (but not between these states and death) are the focus of this study and these prognostic variables will be used to construct models of the relative risks for transitions between each pair of stages. Initially, these factors will be evaluated independently and Hosmer-Lemeshow's criteria for logistic regression of unadjusted $\mathrm{p}<0.25$ will be used to identify candidate predictors for further (adjusted) modelling. ${ }^{75}$ The number of potential predictors included in adjusted models will be limited by the number of transitions providing data for parameter estimates. Peduzzi et al's heuristic for logistic regression of 10 events (and 10 non-events) per parameter estimated will be used to determine the maximum model complexity when identifying predictors for further examination. ${ }^{76}$ If more factors are identified than can be modelled using the Hosmer-Lemeshow screening heuristic, selection will be based on favouring modifiable factors which could be used to inform interventions over non-modifiable factors. Similarly, where multiple predictors are identified that are highly correlated, only one will be retained for further modelling. If data allow estimation of the necessary number of parameters, time-dependent transition probabilities will be estimated in similar ways.

A genetic risk score will be compiled from common variants identified as T2DM-associated from a NZ genomewide association study of T2DM currently in progress. The genetic risk score will be tested for association with the outcomes of the intervention in the sub-sample of participants with genetic information.

\section{Qualitative component}

Inclusion of qualitative approaches in the design and reporting of evaluations of complex health interventions is relatively uncommon and rarely reported, although qualitative components offer opportunities to enhance the interpretation of the quantitative outcome data. ${ }^{77-79}$ The qualitative component of this study will provide important insight into the psychosociocultural factors that influence lifestyle change in relation to regression to normoglycaemia. It will specifically explore the experience of being diagnosed with pre-diabetes and how both those who regress to normoglycaemia at 6 months and those who do not describe the process in relation to barriers to and facilitators of lifestyle changes.

A purposive sample of 60 participants who have completed the 6-month intervention will be sought (30 people who regressed to normoglycaemia and 30 people who did not). The sample will be stratified to include equal numbers of participants by gender and half of the sample will comprise indigenous Māori to ensure that a meaningful level of analysis can be undertaken. Participants will be selected from those who agreed to participate in further research when first recruited into the main study. Those purposively selected will be contacted shortly after completion of the 6-month measures to arrange a face-toface interview if willing to participate in this part of the study. Written consent will be obtained immediately prior to this interview, which will last approximately $60 \mathrm{~min}$. The interviewer (SA), who has, with co-author (DT-L), worked and published extensively in projects where the cross-cultural context is central, will ensure cultural safety throughout the interview process with Māori and Pacific participants. ${ }^{80}{ }^{81}$ With permission, all interviews will be audio recorded and transcribed.

Analysis will be completed by a sub-team (SA, LW, DT-L and KC) using thematic analysis. ${ }^{82}$ This will involve multiple reading of transcriptions followed by the development and description of a priori and emergent themes and concepts. Further analysis will explore interconnections between themes. The thematic findings will also be triangulated with the quantitative data to assist interpretation of results and provide descriptive context. ${ }^{83}$

\section{Economic analysis}

Using the EQ-5D scores, the number and percentage of participants reporting each level of problem on each dimension of the EQ-5D will be described by age, gender and ethnicity, and compared across time and groups (ie, those who regress to normoglycaemia, those who have persistent pre-diabetes and those who progress to T2DM). Participants' self-reported health status as measured on the EQ Visual Analogue Scale will also be reported. An estimate of potential cost savings (acknowledging that any change in glycaemic status cannot be attributed to the programme with certainty) will be calculated from a funder's perspective using average cost estimates and the expected rate of progression to T2DM.

\section{ETHICS AND DISSEMINATION}

Local consultation with Māori was facilitated via Health Hawke's Bay's Operational Māori Advisor and was undertaken at the outset of study planning, particularly in relation to the genetic studies. The study was endorsed by the Priority Population Committee of the regional primary health organisation, Health Hawke's Bay. This committee is tasked with ensuring programmes contribute to equitable access and health gains for Māori, Pacific and other populations living in high deprivation areas (NZ deprivation score 9-10). ${ }^{84}$ Study author, DT-L (Māori public health physician), performed an advisory role across all appropriate planning components of the study and will be in-field cultural advisor across the project, in particular for the research nurse and the qualitative researcher. The study was also endorsed by the University of Otago Ngāi Tahu Research Consultation Committee. 
Further consultation was undertaken specifically with respect to taking blood for genetic analysis. Families will be interested in learning if there is a genetic link with a higher risk of T2DM. The most obvious outcome would include a more responsive health system with early detection, destigmatisation, early 'no blame' intervention and prevention programmes for family who are at risk of developing T2DM. As part of the study, participants will be given a choice on consenting as to the disposal of body fluids / parts as per the University of Otago's and Hawke's Bay District Health Board's Policies and Procedures.

The main study results will be first presented to participating GPs and participating patients. The results of the study will be written and published in peer-reviewed journals and presented at relevant conferences. A written summary of the results will also be distributed to participants, participating GPs, and published on Health Hawke's Bay website for their members.

\section{Author affiliations}

${ }^{1}$ Department of Medicine, University of Otago, Dunedin, New Zealand

${ }^{2}$ Health Hawke's Bay, Hastings, New Zealand

${ }^{3}$ Kaupapa Consulting Ltd, Napier, New Zealand

${ }^{4}$ School of Nursing and Midwifery, Edith Cowan University, Joondalup, Western Australia, Australia

${ }^{5}$ Faculty of Education, Humanities and Health Science, Eastern Institute of Technology, Napier, New Zealand

${ }^{6}$ Centre for Biostatistics, University of Otago, Dunedin, New Zealand

${ }^{7}$ Department of Biochemistry, University of Otago, Dunedin, New Zealand

${ }^{8}$ Department of Preventive and Social Medicine, University of Otago, Dunedin, New Zealand

${ }^{9}$ Department of Medicine, University of Otago Wellington, Wellington, New Zealand

${ }^{10}$ Department of Medicine, University of Colorado, Denver, Colorado, USA

Acknowledgements We acknowledge the commitment of Terrie Spedding, Health Hawke's Bay, the participating primary care practitioners and nurses and the Sport Hawke's Bay Active Living Advisers who contributed to the successful pilot study. We also acknowledge Chris Petersen and Helen Morris, Health Hawke's Bay, who made the necessary requirements to capture study data from the Patient Management System. The pilot study was supported by health service funding from the New Zealand Ministry of Health, a Hawke's Bay Medical Research Foundation grant-in-aid and a New Zealand Society for the Study of Diabetes research award.

Contributors $\mathrm{KC}$ conceived the study. $\mathrm{KC}$ and TF initiated and led the pilot study from the outset. KC, SA, LW, AG, TM, TS, JK and LP contributed to the study design, with DT-L providing cultural guidance. AG wrote the statistical analysis plan and estimated the sample size. KC wrote the first draft of the manuscript with contributions from SA, LW, AG, TM, JK and TS. All authors revised the manuscript for important intellectual content, and read and approved the final version of the manuscript.

Funding The study is funded by the Health Research Council of New Zealand project grant (16/344). This grant is administered by the University of Otago, who employs KC, AG, TM, TS and JK. SA and TF are supported in part by this research grant through sub-contracts.

Competing interests TF is employed by Health Hawke's Bay who co-ordinates the general practitioners participating in the research. JK reports receiving personal fees for consulting or speaking from MSD and Novartis. LP reports receiving personal fees for consulting or speaking from Novo Nordisk, Sanofi, Astra Zeneca, Boehringer-Ingelheim, Merck, Janssen, WebMD, Medscape and UpToDate.

Patient consent for publication Not required.

Ethics approval This study has received ethics approval from the Northern A Health and Disability Ethics Committee, New Zealand (Ethics Reference: 17/ NTA/24). The study is registered with the Australian New Zealand Clinical Trials Registry (ACTRN12617000591358).

Provenance and peer review Not commissioned; externally peer reviewed.
Open access This is an open access article distributed in accordance with the Creative Commons Attribution Non Commercial (CC BY-NC 4.0) license, which permits others to distribute, remix, adapt, build upon this work non-commercially, and license their derivative works on different terms, provided the original work is properly cited, appropriate credit is given, any changes made indicated, and the use is non-commercial. See: http://creativecommons.org/licenses/by-nc/4.0/.

ORCID iD

Kirsten Coppell http://orcid.org/0000-0003-0996-2874

\section{REFERENCES}

1 Danaei G, Finucane MM, Lu Y, et al. National, regional, and global trends in fasting plasma glucose and diabetes prevalence since 1980: systematic analysis of health examination surveys and epidemiological studies with 370 country-years and 2.7 million participants. Lancet 2011;378:31-40.

2 Vos T, Barber RM, Bell B, et al. Global, regional, and national incidence, prevalence, and years lived with disability for 301 acute and chronic diseases and injuries in 188 countries, 1990-2013: a systematic analysis for the global burden of disease study 2013. Lancet 2015;386:743-800.10.1016/S0140-6736(15)60692-4

3 American Diabetes Association. Economic costs of diabetes in the U.S. in 2007. Diabetes Care 2008;31:596-615. Erratum in: Diabetes Care 2008;31:1271.10.2337/dc08-9017

4 American Diabetes Association. Economic costs of diabetes in the U.S. in 2017. Diabetes Care 2018;41:917-28.10.2337/dci18-0007

5 Ogurtsova K, da Rocha Fernandes JD, Huang Y, et al. IDF diabetes atlas: global estimates for the prevalence of diabetes for 2015 and 2040. Diabetes Res Clin Pract 2017;128:40-50.10.1016/j. diabres.2017.03.024

6 Ali MK, Bullard KM, Saydah S, et al. Cardiovascular and renal burdens of prediabetes in the USA: analysis of data from serial cross-sectional surveys, 1988-2014. Lancet Diabetes Endocrinol 2018;6:392-403.10.1016/S2213-8587(18)30027-5

7 Coppell KJ, Mann JI, Williams SM, et al. Prevalence of diagnosed and undiagnosed diabetes and prediabetes in New Zealand: findings from the 2008/09 adult nutrition survey. N Z Med J 2013;126:23-42.

8 Lee AK, Warren B, Liu C, et al. Number and characteristics of US adults meeting prediabetes criteria for diabetes prevention programs: NHANES 2007-2016. J Gen Intern Med (Published Online First: 11 March 2019).

9 Song $\mathrm{Y}$, Zhang $\mathrm{X}$, Zhang $\mathrm{H}$, et al. Prevalence of diabetes and prediabetes in adults from a third-tier city in eastern China: a crosssectional study. Diabetes Ther 2019;10:1473-85.10.1007/s13300019-0655-X

10 Eades CE, Leese GP, Evans JMM. Incidence of impaired glucose regulation and progression to type 2 diabetes mellitus in the Tayside region of Scotland. Diabetes Res Clin Pract 2014;104:e16-19.10.1 016/j.diabres.2014.01.012

11 Tabák AG, Herder C, Rathmann W, et al. Prediabetes: a high-risk state for diabetes development. Lancet 2012;379:2279-90.10.1016/ S0140-6736(12)60283-9

12 Knowler WC, Barrett-Connor E, Fowler SE, et al. Reduction in the incidence of type 2 diabetes with lifestyle intervention or metformin. N Engl J Med 2002;346:393-403.

13 Kosaka K, Noda M, Kuzuya T. Prevention of type 2 diabetes by lifestyle intervention: a Japanese trial in IGT males. Diabetes Res Clin Pract 2005;67:152-62.10.1016/j.diabres.2004.06.010

14 Lindahl B, Nilssön TK, Borch-Johnsen K, et al. A randomized lifestyle intervention with 5-year follow-up in subjects with impaired glucose tolerance: pronounced short-term impact but long-term adherence problems. Scand J Public Health 2009;37:434-42. Erratum in: Scand J Public Health 2009;37:443.10.1177/1403494808101373

15 Tuomilehto J, Lindström J, Eriksson JG, et al. Prevention of type 2 diabetes mellitus by changes in lifestyle among subjects with impaired glucose tolerance. N Engl J Med 2001;344:1343-50.10.1056/NEJM200105033441801

16 Pan XR, Li GW, Hu YH, et al. Effects of diet and exercise in preventing NIDDM in people with impaired glucose tolerance. The dA Qing IGT and diabetes study. Diabetes Care 1997;20:537-44.

17 Ramachandran A, Snehalatha C, Mary S, et al. The Indian diabetes prevention programme shows that lifestyle modification and metformin prevent type 2 diabetes in Asian Indian subjects with impaired glucose tolerance (IDPP-1). Diabetologia 2006;49:289-97.10.1007/s00125-005-0097-z

18 Hamman RF, Horton E, Barrett-Connor E, et al. Factors affecting the decline in incidence of diabetes in the diabetes prevention program outcomes study (DPPOS). Diabetes 2015;64:989-98. 
19 Gillies CL, Abrams KR, Lambert PC, et al. Pharmacological and lifestyle interventions to prevent or delay type 2 diabetes in people with impaired glucose tolerance: systematic review and metaanalysis. BMJ 2007:334.10.1136/bmj.39063.689375.55

20 Stevens JW, Khunti K, Harvey R, et al. Preventing the progression to type 2 diabetes mellitus in adults at high risk: a systematic review and network meta-analysis of lifestyle, pharmacological and surgical interventions. Diabetes Res Clin Pract 2015;107:320-31.10.1016/j. diabres.2015.01.027

21 Yakubovich N, Gerstein HC. Is regression to normoglycaemia clinically important? Lancet 2012;379:2216-8.10.1016/S01406736(12)60828-9

22 Ard J. Obesity in the US: what is the best role for primary care? BMJ 2015;350:g7846.10.1136/bmj.g7846

23 Cefalu WT. Steps toward the meaningful translation of prevention strategies for type 2 diabetes. Diabetes Care 2012;35:663-5.10.2337/ dc12-0119

24 Kahan S, Manson JE. Nutrition counseling in clinical practice: how clinicians can do better. JAMA 2017;318:1101-2.

25 Garfield SA, Malozowski S, Chin MH, et al. Considerations for diabetes translational research in real-world settings. Diabetes Care 2003;26:2670-4.10.2337/diacare.26.9.2670

26 Eddy DM, Schlessinger L, Kahn R. Clinical outcomes and costeffectiveness of strategies for managing people at high risk for diabetes. Ann Intern Med 2005;143:251-64.10.7326/0003-4819-1434-200508160-00006

27 Hernan WH, Brandle M, Zhang P, et al. Costs associated with the primary prevention of type 2 diabetes mellitus in the diabetes prevention program. Diabetes Care 2003;26:36-47.

28 Bo S, Ciccone G, Baldi C, et al. Effectiveness of a lifestyle intervention on metabolic syndrome. A randomized controlled trial. $J$ Gen Intern Med 2007;22:1695-703.10.1007/s11606-007-0399-6

29 Sakane N, Sato J, Tsushita K, et al. Japan diabetes prevention program (JDPP) Research Group. prevention of type 2 diabetes in a primary healthcare setting: three-year results of lifestyle intervention in Japanese subjects with impaired glucose tolerance. BMC Public Health 2011;11:40.

30 Absetz P, Valve R, Oldenburg B, et al. Type 2 Diabetes Prevention in the "Real World": One-year results of the GOAL Implementation Trial. Diabetes Care 2007;30:2465-70.10.2337/dc07-0171

31 Adachi M, Yamaoka K, Watanabe M, et al. Effects of lifestyle education program for type 2 diabetes patients in clinics: study design of a cluster randomized trial. BMC Public Health 2010;10:742.10.1186/1471-2458-10-742

32 Kilkkinen A, Heistaro S, Laatikainen T, et al. Prevention of type 2 diabetes in a primary health care setting. interim results from the greater green triangle (GGT) diabetes prevention project. Diabetes Res Clin Pract 2007;76:460-2.

33 Sacerdote C, Fiorini L, Rosato R, et al. Randomized controlled trial: effect of nutritional counselling in general practice. Int J Epidemiol 2006;35:409-15.10.1093/ije/dyi170

34 Vadstrup ES, Frølich A, Perrild H, et al. Lifestyle intervention for type 2 diabetes patients - trial protocol of the Copenhagen type 2 diabetes rehabilitation project. BMC Public Health 2009:9:166.10.1186/1471-2458-9-166

35 Vadstrup ES, Frølich A, Perrild H, et al. Effect of a group-based rehabilitation programme on glycaemic control and cardiovascular risk factors in type 2 diabetes patients: the Copenhagen type 2 diabetes rehabilitation project. Patient Educ Couns 2011;84:185-90.10.1016/j.pec.2010.06.031

36 Ashenden R, Silagy C, Weller D. A systematic review of the effectiveness of promoting lifestyle change in general practice. Fam Pract 1997;14:160-76.10.1093/fampra/14.2.160

37 Chan BC, Jayasinghe UW, Christl B, et al. The impact of a team-based intervention on the lifestyle risk factor management practices of community nurses: outcomes of the community nursing SNAP trial. BMC Health Serv Res 2013:13:54.10.1186/1472-6963-13-54

38 Neil HAW, Roe L, Godlee RJP, et al. Randomised trial of lipid lowering dietary advice in general practice: the effects on serum lipids, lipoproteins, and antioxidants. BMJ 1995;310:569-73.10.1136/ bmi.310.6979.569

39 Wadden TA, Volger S, Sarwer DB, et al. A two-year randomized trial of obesity treatment in primary care practice. $N$ Engl J Med 2011;365:1969-79.10.1056/NEJMoa1109220

40 Kushner RF. Barriers to providing nutrition counseling by physicians: a survey of primary care practitioners. Prev Med 1995;24:546-52.10.1006/pmed.1995.1087

41 Huang Y, Cai X, Mai W, et al. Association between prediabetes and risk of cardiovascular disease and all cause mortality: systematic review and meta-analysis. BMJ 2016;355.10.1136/bmj.i5953
42 Perreault L, Pan Q, Schroeder EB, et al. Regression from prediabetes to normal glucose regulation and prevalence of microvascular disease in the diabetes prevention program outcomes study (DPPOS). Diabetes Care 2019;42:1809-15.

43 Perreault L, Færch K, pre-diabetes A. Approaching prediabetes. J Diabetes Complications 2014;28:226-33.10.1016/j. jdiacomp.2013.10.008

44 Perreault L, Pan Q, Mather KJ, et al. Effect of regression from prediabetes to normal glucose regulation on long-term reduction in diabetes risk: results from the diabetes prevention program outcomes study. Lancet 2012;379:2243-51.10.1016/S01406736(12)60525-X

45 Perreault L, Kahn SE, Christophi CA, et al. Diabetes prevention program Research Group. regression from pre-diabetes to normal glucose regulation in the diabetes prevention program. Diabetes Care 2009;32:1583-8.

46 Maruthur NM, Ma Y, Delahanty LM, et al. Diabetes prevention program Research Group. early response to preventive strategies in the diabetes prevention program. $J$ Gen Intern Med 2013;28:1629-36.

47 Nanditha A, Ram J, Snehalatha C, et al. Early improvement predicts reduced risk of incident diabetes and improved cardiovascular risk in prediabetic Asian Indian men participating in a 2-year lifestyle intervention program. Diabetes Care 2014;37:3009-15.10.2337/ dc14-0407

48 Coppell KJ, Abel SL, Freer T, et al. The effectiveness of a primary care nursing-led dietary intervention for prediabetes: a mixed methods pilot study. BMC Fam Pract 2017;18:106.10.1186/s12875017-0671-8

49 Sussman JB, Kent DM, Nelson JP, et al. Improving diabetes prevention with benefit based tailored treatment: risk based reanalysis of Diabetes Prevention Program. BMJ 2015;350:h454.10.1136/bmj.h454

50 Eccles M, Grimshaw J, Campbell M. Research designs for studies evaluating the effectiveness of change and improvement strategies. Qual Saf Health Care 2003;12:47-52.10.1136/qhc.12.1.47

51 Shojania KG, Grimshaw JM. Evidence-Based quality improvement: the state of the science. Health Aff 2005;24:138-50.10.1377/ hlthaff.24.1.138

52 Ministry of Health. New Zealand primary care Handbook 2012 (updated 2013): cardiovascular disease risk assessment. Wellington: Ministry of Health, 2013.

53 Statistics New Zealand. 2013 census QuickStats about a place, 2013. Available: http://archive.stats.govt.nz/Census/2013-census/ profile-and-summary-reports/quickstats-about-a-place [Accessed 30 Jul 2019].

54 Statistics New Zealand. 2013 census QuickStats about Māori, 2013. Available: http://archive.stats.govt.nz/Census/2013-census/ profile-and-summary-reports/quickstats-about-maori-english.aspx [Accessed 30 Jul 2019].

55 Paxton A, Strycker L, Toobert D, et al. Starting the conversation performance of a brief dietary assessment and intervention tool for health professionals. Am J Prev Med 2011;40:67-71.

56 Ministry of Health. Be active every day: physical activity for adults. Wellington: Ministry of health, 2015. Available: https://www.healthed. govt.nz/resource/be-active-every-day-physical-activity-adults [Accessed $30 \mathrm{Jul}$ 2019].

57 Lorig KR, Ritter P, Stewart AL, et al. Chronic disease selfmanagement program: 2-year health status and health care utilization outcomes. Med Care 2001:39:1217-23.

58 Lorig KR, Sobel DS, Stewart AL, et al. Evidence suggesting that a chronic disease self-management program can improve health status while reducing hospitalization: a randomized trial. Med Care 1999;37:5-14.

59 Diabetes New Zealand. Diabetes and healthy food choices. Wellington: diabetes New Zealand Inc, 2019. Available: https://www. diabetes.org.nz/pamphlet-ordering [Accessed $30 \mathrm{Jul}$ 2019].

60 Coppell KJ, Kataoka M, Williams SM, et al. Nutritional intervention in patients with type 2 diabetes who are hyperglycaemic despite optimised drug treatment-Lifestyle Over and Above Drugs in Diabetes (LOADD) study: randomised controlled trial. BMJ 2010;341:c3337.

61 Sallis JF, Grossman RM, Pinski RB, et al. The development of scales to measure social support for diet and exercise behaviors. Prev Med 1987;16:825-36.10.1016/0091-7435(87)90022-3

62 Sarason IG, Levine HM, Basham RB, et al. Assessing social support: the social support questionnaire. J Pers Soc Psychol 1983:44:127-39.10.1037/0022-3514.44.1.127

63 Buysse DJ, Reynolds CF, Monk TH, et al. The Pittsburgh sleep quality index: a new instrument for psychiatric practice and research. Psychiatry Res 1989;28:193-213.10.1016/0165-1781(89)90047-4 
64 Andrés A, Saldaña C, Gómez-Benito J. Establishing the stages and processes of change for weight loss by consensus of experts. Obesity 2009;17:1717-23.10.1038/oby.2009.100

65 Ceccarini M, Borrello M, Pietrabissa G, et al. Assessing motivation and readiness to change for weight management and control: an in-depth evaluation of three sets of instruments. Front Psychol 2015;6:511.10.3389/fpsyg.2015.00511

66 Craig CL, Marshall AL, Sjostrom M, et al. International physical activity questionnaire: 12-country reliability and validity. Med Sci Sports Exerc 2003;35:1381-95.10.1249/01.MSS.0000078924.61453. FB

67 EuroQol Group. EuroQol-a new facility for the measurement of health-related quality of life. Health Policy 1990;16:199-208.10.1016/0168-8510(90)90421-9

68 Glasziou P, Alexander J, Beller E, et al. Which health-related quality of life score? A comparison of alternative utility measures in patients with Type 2 diabetes in the ADVANCE trial. Health Qual Life Outcomes 2007:5:21.10.1186/1477-7525-5-21

69 Matthews DR, Hosker JP, Rudenski AS, et al. Homeostasis model assessment: insulin resistance and beta-cell function from fasting plasma glucose and insulin concentrations in man. Diabetologia 1985;28:412-9.10.1007/BF00280883

70 McAuley KA, Williams SM, Mann JI, et al. Diagnosing insulin resistance in the general population. Diabetes Care 2001;24:460-4.10.2337/diacare.24.3.460

71 Kawamori R, Tajima N, Iwamoto Y, et al. Voglibose for prevention of type 2 diabetes mellitus: a randomised, double-blind trial in Japanese individuals with impaired glucose tolerance. Lancet 2009;373:1607-14.10.1016/S0140-6736(09)60222-1

72 Elm Evon, Altman DG, Egger M, et al. Strengthening the reporting of observational studies in epidemiology (STROBE) statement: guidelines for reporting observational studies. BMJ 2007;335:806-8.10.1136/bmj.39335.541782.AD

73 Collins GS, Reitsma JB, Altman DG, et al. Transparent reporting of a multivariable prediction model for individual prognosis or diagnosis
(TRIPOD): the TRIPOD statement. BMJ 2014;350:g7594.10.1136/ bmj.g7594

74 Froot KA. Consistent covariance matrix estimation with crosssectional dependence and heteroskedasticity in financial data. The Journal of Financial and Quantitative Analysis 1989;24:333-55.

75 Hosmer D, Lemeshow S. Applied logistic regression. 2nd Edition. New York: John Wiley \& Sons, 2000.

76 Peduzzi P, Concato J, Kemper E, et al. A simulation study of the number of events per variable in logistic regression analysis. J Clin Epidemiol 1996;49:1373-9.10.1016/S0895-4356(96)00236-3

77 Lewin S, Glenton C, Oxman AD. Use of qualitative methods alongside randomised controlled trials of complex healthcare interventions: methodological study. BMJ 2009;339:b3496.10.1136/ bmj.b3496

78 Oakley A, Strange V, Bonell C, et al. Process evaluation in randomised controlled trials of complex interventions. BMJ 2006;332:413-6.10.1136/bmj.332.7538.413

79 O'Cathain A, Thomas KJ, Drabble SJ, et al. What can qualitative research do for randomised controlled trials? A systematic mapping review. BMJ Open 2013;3:e002889.10.1136/ bmjopen-2013-002889

80 Abel S, Tipene-Leach D. SUDI prevention: a review of Maori safe sleep innovations for infants. N Z Med J 2013;126:86-94.

81 Tipene-Leach DC, Coppell KJ, Abel S, et al. Ngāti and healthy: translating diabetes prevention evidence into community action. Ethn Health 2013;18:402-14.10.1080/13557858.2012.754406

82 Braun V, Clarke V. Using thematic analysis in psychology. Qual Res Psychol 2006;3:77-101.10.1191/1478088706qp063oa

83 Pope C, Mays N. Qualitative methods in health research. In: Pope C, Mays N, eds. Qualitative research in health care. 3rd ed. Massachusetts: Blackwell, 2006.

84 Atkinson J, Salmond C, Crampton P. NZDep2013 index of deprivation. Wellington: Department of Public Health, University of Otago Wellington, 2014. 